\title{
O papel do enfermeiro do ambulatório de prevenção e tratamento da síndrome mão- pé induzida pelo uso de Capecitabina
}

\author{
The role of the nurse in the ambulatory for the prevention and treatment of hand-foot syndrome \\ induced by the use of Capecitabine \\ El papel de la enfermera en el ambulatorio para la prevención y tratamiento del síndrome mano- \\ pie inducido por el uso de Capecitabina
}

Recebido: 28/03/2021 | Revisado: 04/04/2021 | Aceito: 09/04/2021 | Publicado: 19/04/2021

Franciele Malhard de Arruda

ORCID: https://orcid.org/0000-0002-5168-3628 Instituto Nacional de Câncer, Brasil

E-mail:francielemalhard@gmail.com

Lucimere Maria dos Santos

ORCID: https://orcid.org/0000-0003-3455-1268 Instituto Nacional de Câncer, Brasil

E-mail: lucimereuff@gmail.com

Milena Quaresma Lopes

ORCID: https://orcid.org/0000-0002-4814-3444 Instituto Nacional de Câncer, Brasil

E-mail: milenaq_lopes@ hotmail.com

Livia Maria Pesco Bitencourt

ORCID: https://orcid.org/0000-0002-0406-4357 Instituto Nacional de Câncer, Brasil E-mail: liviabit@gmail.com

Shirlei da Silva Rodrigues

ORCID: https://orcid.org/0000-0002-6074-471X Instituto Nacional de Câncer, Brasil

E-mail: shsferreira@gmail.com

Raquel de Souza Ramos

ORCID: https://orcid.org/0000-0003-1939-7864 Instituto Nacional de Câncer, Brasil E-mail:kakelramos@gmail.com

\begin{abstract}
Resumo
Introdução: A eritrodisestesia palmo-plantar, também conhecida como Síndrome Mão-Pé (SMP), é uma reação adversa causada pela terapia antineoplásica oral Capecitabina que causa impacto na qualidade de vida dos pacientes com esse tratamento quimioterápico. O profissional enfermeiro tem como instrumento de cuidado a consulta de enfermagem que possibilita uma intervenção educativa sistematizada, fundamental para composição de um cuidado individualizado e integral. Objetivo: Analisar as ações desenvolvidas pelo enfermeiro no ambulatório de prevenção e tratamento da Síndrome Mão-Pé induzida pelo uso de Capecitabina. Metodologia: Estudo descritivo, com abordagem qualitativa e observação participante, realizado em um instituto de referência em oncologia, situado no Estado do Rio de Janeiro, no período de outubro a novembro de 2020. Foi elaborado um diário de campo a partir dos dados referentes às ações desenvolvidas pelo profissional durante a consulta de enfermagem, o fluxo percorrido pelos pacientes atendidos nesse ambulatório, as anotações do enfermeiro no livro de registro dos pacientes e impressões da pesquisadora. Resultados: Após a análise criteriosa dos dados emergiram duas categorias de análise: ações administrativas e ações técnicas e educativas. Conclusão: Identificou-se que o fluxo seguido oportuniza o acompanhamento dos pacientes em uso de antineoplásico oral Capecitabina em sua quase totalidade. As consultas de enfermagem são operacionalizadas com intuito preventivo da instauração da síndrome em um importante processo de interação/integração com o paciente, utilizando-se a educação em saúde como estratégia de cuidado para prevenção e manejo da síndrome, contribuindo para disseminação de conhecimento acerca do processo de trabalho do enfermeiro em ambulatório especializado e contemporâneo.
\end{abstract}

Palavras-chave: Síndrome mão-pé; Capecitabina; Cuidados de enfermagem; Enfermagem. 


\begin{abstract}
Introduction: Palmoplantar erythrodysesthesia, also known as hand-foot syndrome (PMS), is an adverse reaction caused by the oral antineoplastic therapy Capecitabine that causes the greatest impact on the quality of life of patients with this chemotherapy treatment. The professional nurse has the nursing consultation as an instrument of care, which enables a systematic and permanent educational intervention fundamental to the composition of individualized and comprehensive care. General Objective: Analyze the actions developed by nurses in the outpatient prevention and treatment of Hand-Foot Syndrome induced by the use of Capecitabine. Methodology: Descriptive study, with a qualitative approach and participant observation, carried out in a reference institute in oncology, located in the State of Rio de Janeiro, from October to November 2020. A field diary was prepared based on data referring to the actions taken by the professional during the nursing consultation, the flow of patients seen in this clinic, the nurse's notes in the patient record book, and the researcher's impressions. Results: After careful analysis of the data, two categories of analysis emerged: administrative actions and technical and educational actions. Conclusion: It was found that the flow followed provides an opportunity for the follow-up of patients using Capecitabine oral antineoplastic in almost its entirety. Nursing consultations are carried out with the preventive intention of establishing the syndrome in an important process of interaction / integration with the patient, using health education as a care strategy for the prevention and management of the syndrome, contributing to the dissemination of knowledge about the nurses' work process in a specialized and contemporary outpatient clinic.
\end{abstract}

Keywords: Hand-footsyndrome; Capecitabine; Nursing care; Nursing.

\title{
Resumen
}

Introducción: La eritrodisestesia palmoplantar, conocida popularmente como síndrome mano-pie (SPM), es un efecto adverso provocado por la terapia antineoplásica oral Capecitabina que provoca un mayor impacto en la calidad de vida de estos pacientes. El enfermero profesional tiene la consulta de enfermería como un instrumento asistencial que permite una intervención educativa sistemática, fundamental para la composición de la atención individualizada e integral. Objetivo: Analizar las acciones desarrolladas por enfermeras en la prevención y tratamiento ambulatorio del Síndrome Mano-Pie inducido por el uso de Capecitabina. Metodología: Estudio descriptivo, con abordaje cualitativo y observación participante, realizado en un instituto de referencia en oncología, ubicado en el Estado de Rio de Janeiro, de octubre a noviembre de 2020. Se elaboró un diario de campo a partir de los datos relacionados con las acciones desarrolladas por el profesional durante la consulta de enfermería, el flujo cubierto por los pacientes atendidos en esta clínica, las notas de la enfermera en el libro de registro del paciente y las impresiones del investigador. Resultados: Después de un análisis cuidadoso de los datos, surgieron dos categorías de análisis: acciones administrativas y acciones técnicas y educativas. Conclusión: Se encontró que el flujo seguido brinda una oportunidad para el seguimiento de los pacientes que utilizan el antineoplásico oral Capecitabina en casi su totalidad. Las consultas de enfermería se realizan con la intención preventiva de establecer el síndrome en un importante proceso de interacción / integración con el paciente, utilizando la educación en salud como estrategia asistencial para la prevención y manejo del síndrome, contribuyendo a la difusión del conocimiento sobre las enfermeras. 'proceso de trabajo en una clínica ambulatoria especializada y contemporánea.

Palabras clave: Síndrome mano-pie; Capecitabina; Atención de enfermería; Enfermería.

\section{Introdução}

A Organização Mundial da Saúde (OMS) delimita a ocorrência de 27 milhões de novos casos de câncer que afetarão a população mundial. Estima-se que haverá 17 milhões de mortes por câncer. Assim, pode-se caracterizar esta patologia como o principal problema de saúde pública no mundo (Brasil, 2018).

O Ministério da Saúde (MS) delimita estruturalmente o câncer como: "perda do controle de divisão celular e capacidade de invadir outras estruturas orgânicas", sendo concebido como um transtorno funcional (Brasil, 2019b). Partindo desse princípio são estabelecidas políticas públicas que visam prevenção, controle e tratamento desta afecção.

Dentre as diferentes técnicas e abordagens escolhidas para o tratamento oncológico existe a quimioterapia, que consiste em um tratamento sistêmico para o câncer por meio de medicamentos denominados antineoplásicos, administrados em intervalos regulares. Sua administração pode ser efetuada por várias vias, como: endovenosa, intratecal, tópica, dentre outras. Para esse estudo, enfatiza-se a terapêutica através do uso da Capecitabina que é uma terapia realizada por via oral, oferta terapêutica em ascendimento que perpassa a caracterização de tratamento quimioterápico restrito a administração intravenosa, configurando-se menos invasivo (Brasil, 2019; Oliveira \& Queiroz, 2012; Demoliner \& Corte, 2008). 
A Capecitabina é uma das possibilidades de terapia antineoplásica oral. Sua composição deriva do carbamato de fluoropirimidina, configurado como pró-droga sistêmica do 5-fluorouracil (5-FU). Trata-se de um agente citotóxico de tumor ativado e tumor seletivo, desenvolvido para administração oral, indicado como monoterapia, terapia combinada para tratamento de câncer de mama metastático, tratamento adjuvante para câncer colorretal, terapia combinada para colorretal metastático e câncer gástrico. Dentre as toxicidades referente a utilização de Capecitabina/Xeloda ${ }^{\circledR}$ identifica-se a eritrodisestesia palmo-plantar, conhecida como Síndrome Mão-Pé (SMP) (Capecitabina, 2020; Junior et al., 2017).

A eritrodisestesia palmo-plantar é caracterizada como uma reação cutânea frequente ocasionada pela quimioterapia antineoplásica configurando-se como um importante problema clínico ao indivíduo com neoplasia maligna em uso desta terapêutica (Costa et al., 2018). Esta Síndrome é classificada em três graus de acordo com a Terminologia Comum para Critérios de Eventos Adversos (CTCAE). No primeiro grau ocorrem alterações discretas na pele ou dermatite dolorosa manifestada através de eritema, edema ou hiperceratose. Quando se estabelece o segundo grau, a sintomatologia definidora são alterações cutâneas correlacionadas

à dor, como flictema, descamação, lesões, edema ou hiperqueratose. É constatada a elevação para o terceiro grau quando ocorrem alterações cutâneas drásticas associadas a dor que progride para restrição do autocuidado (Sobrafo, 2011), sendo indicativo nesses casos a redução ou a suspensão do tratamento (Ema, 2015).

Considerando a complexidade do manejo dessa síndrome, pontua-se a imprescindibilidade do cuidado desempenhado pelo profissional enfermeiro por meio da consulta de enfermagem estruturada como um instrumento de cuidado fundamental nesse processo para composição de uma assistência individualizada e integral, sendo uma atuação privativa do enfermeiro regulamentada através da Resolução do Conselho Federal de Enfermagem (COFEN) de número 358, do ano de 2009, que a instrumentaliza como integrante do Processo de Enfermagem (PE), instrumento intelectual de trabalho do profissional enfermeiro (Cofen, 2009; Barros et al., 2015; Santos et al., 2020).

A consulta de enfermagem implementada em ambiente ambulatorial especializado se apresenta como ação inovadora do cuidado, favorecendo o encadeamento de condições de saúde específicas e habilitação profissional em intervenção clínica, sem demandar internação (Canonici, 2014).

\section{Objetivo}

Analisar as ações desenvolvidas pelo enfermeiro no ambulatório de prevenção e tratamento da Síndrome Mão-Pé induzida pelo uso de Capecitabina em um instituto de referência em oncologia do Rio de Janeiro.

\section{Metodologia}

Estudo descritivo de abordagem qualitativa do tipo pesquisa de campo. O estudo se desenvolveu no ambulatório de prevenção e tratamento da síndrome mão-pé de um hospital referência em oncologia no rio de janeiro.

A pesquisa foi submetida e aprovada pelo Comitê de Ética em Pesquisa da instituição referida sob o número do parecer CAAE 37792320.0.0000.5274. Foram assegurados o sigilo e a confidencialidade dos dados, em consonância com a Resolução $n^{\circ}$ 510/16 (Brasil, 2016), do Conselho Nacional de Saúde (CNS), que regulamenta as diretrizes envolvendo a pesquisa com seres humanos no Brasil.

Foi esclarecido à participante da pesquisa quanto aos objetivos e a metodologia do estudo, bem como da apresentação do Termo de Consentimento Livre e Esclarecido (TCLE), de acordo com o modelo utilizado pela instituição.

A técnica utilizada para a coleta de dados foi a observação participativa. Trata-se de uma técnica para conseguir informações utilizando os sentidos na obtenção de determinados aspectos da realidade, não consistindo apenas em ver e ouvir, mas também em examinar fatos e fenômenos que se deseje estudar (Marconi \& Lakatos, 2017). 
Uma vez aprovada a pesquisa, procedeu-se o agendamento dos encontros com o enfermeiro responsável pelo ambulatório de prevenção e tratamento da Síndrome Mão-Pé. Houve um total de cinco encontros que viabilizaram a coleta de dados nos meses de outubro e novembro, sendo três encontros em horário das 13 às 16 horas e dois em horário integral de funcionamento, das 08 às 16 horas.

A coleta de dados ocorreu em cinco etapas que proporcionou a elaboração de um diário de campo para descrição dos dados na íntegra. O diário de campo elaborado foi constituído pelas impressões do pesquisador, descrição das ações desenvolvidas pelo profissional durante a consulta de enfermagem, bem como identificação do fluxo percorrido pelos pacientes atendidos nesse ambulatório.

Os dados foram lidos exaustivamente e em seguida analisados de forma criteriosa e em profundidade, o que permitiu identificar dois conjuntos de ações desenvolvidas pelo profissional enfermeiro: ações administrativas e ações técnicas e educativas.

Quanto aos critérios de inclusão e exclusão não se aplicou ao estudo, uma vez que neste ambulatório apenas um enfermeiro desempenha função.

\section{Resultados}

A análise dos dados permitiu identificar que existe um fluxo de atendimento para os pacientes atendidos nessa instituição até que eles cheguem ao ambulatório de prevenção e tratamento da Síndrome Mão-Pé. Os pacientes dão entrada nessa instituição sendo atendidos inicialmente pela clínica, de acordo com a localização anatômica do tumor após diagnóstico histopatológico. Esse fluxo encontra-se representado na Figura 1.

Figura 1 - Fluxo de atendimento ao paciente assistido pelo Ambulatório da Síndrome Mão-Pé.

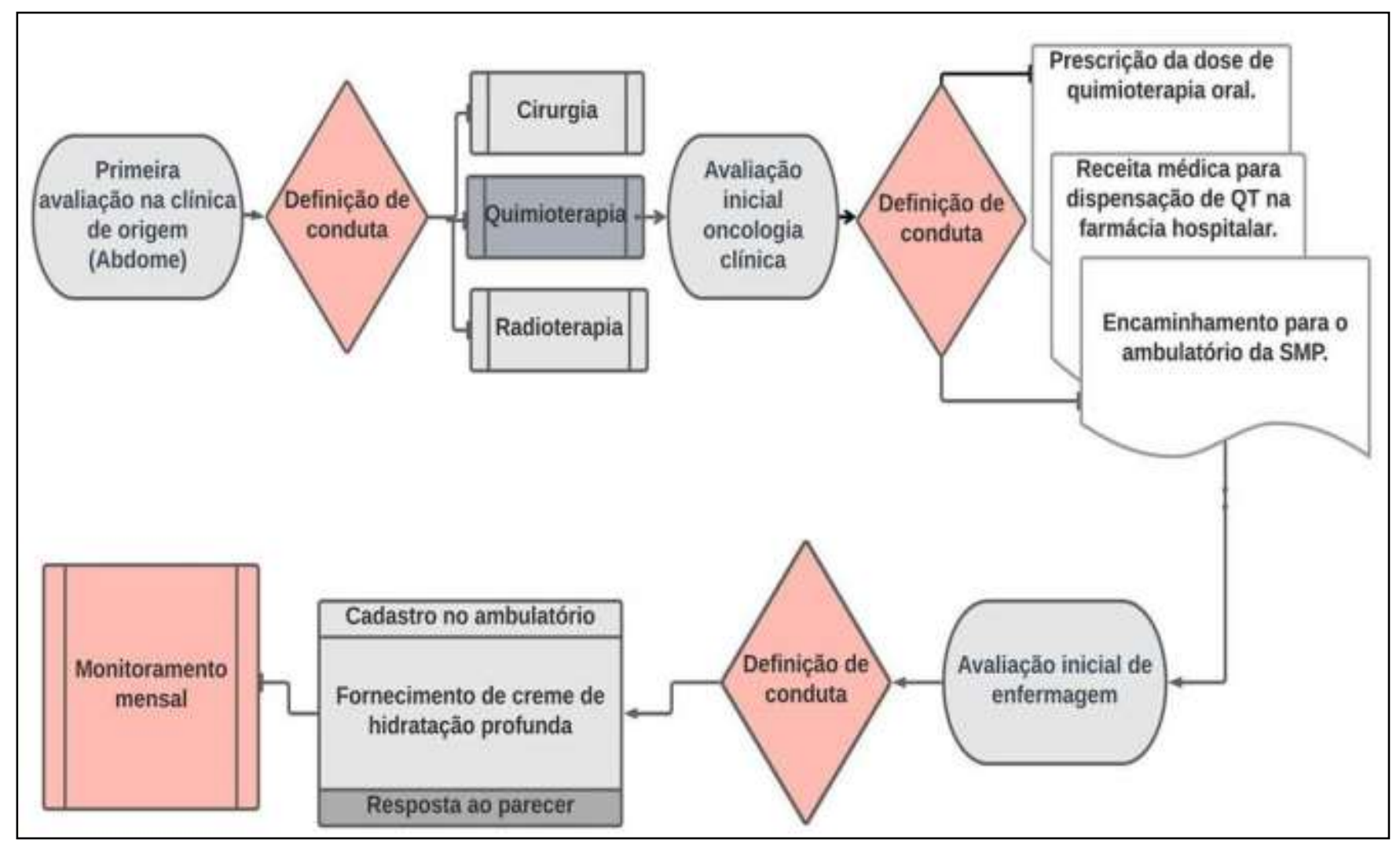

Fonte: Autores (2020). 
Nessa primeira avaliação feita pela clínica inicial faz-se uma análise sobre qual a melhor proposta terapêutica. Havendo indicação de tratamento clínico, o paciente é encaminhado para avaliação da oncologia clínica, essa por sua vez define qual a melhor proposta clínica a seguir. Sendo prescrito tratamento com quimioterápico antineoplásico oral Capecitabina, os pacientes recebem a receita médica, são encaminhados à farmácia hospitalar para a retirada do fármaco e encaminhados para atendimento especializado de enfermagem no ambulatório de prevenção e tratamento da síndrome mão-pé.

A análise dos registros compilados no diário de campo permitiu a compreensão das ações desenvolvidas pelo enfermeiro no ambulatório de prevenção e tratamento da Síndrome Mão-Pé, reconhecida como ações administrativas e ações técnicas e educativas. Visando a organização dos dados, procedeu-se à elaboração de quadros apresentados no decorrer deste texto, onde é possível identificar as ações que caracterizam cada conjunto de ações.

\section{Ações Administrativas}

Os momentos de observação participante propiciaram a identificação de diversas ações de caráter administrativo. Foi ainda possível constatar que estas ações, por vezes, envolvem o atendimento das necessidades de outros setores adjacentes ao ambulatório de prevenção e tratamento da Síndrome Mão-Pé.

Vaghetti et al. (2004) ressalta que as ações administrativas se configuram como integrante da dinâmica de serviço do enfermeiro, assegurando dessa forma a organização institucional e o direcionamento das atividades por meio de ações que são embasadas nas competências em administração e gerenciamento desse profissional. Segundo Vaghetti et al. (2004) o enfermeiro é ainda responsável por gerir "recursos físicos, capital humano, materiais e informações a respeito de sua prática assistencial." Apresenta-se esse tipo de ações no Quadro 1. 
Quadro 1 - Ações administrativas desenvolvidas pelo Enfermeiro do Ambulatório de prevenção e tratamento da Síndrome MãoPé

\begin{tabular}{|c|c|}
\hline \multicolumn{2}{|c|}{ Ações administrativas desenvolvidas no ambulatório SMP } \\
\hline Ação & Característica da ação \\
\hline Identificação do paciente no setor & $\begin{array}{l}\text { Registro dos dados do paciente em livro próprio do setor e em planilhas do } \\
\text { Microsoft Excel® para identificação do paciente no ambulatório. }\end{array}$ \\
\hline Marcação de consulta & Agendamento das consultas de enfermagem subsequentes. \\
\hline Busca ativa de pacientes faltosos & $\begin{array}{l}\text { Levantamento dos pacientes faltosos; } \\
\text { Contato telefônico para reagendamento da consulta de enfermagem em caso de falta } \\
\text { à consulta agendada. }\end{array}$ \\
\hline $\begin{array}{l}\text { Monitoramento de } \\
\text { internados (Contato feito pelo } \\
\text { paciente e/ou familiar) }\end{array}$ & $\begin{array}{l}\text { Armazenamento de informações referente à clínica, leito e agendamento de } \\
\text { consulta; } \\
\text { Avaliação do paciente e distribuição do hidratante no setor de internação. }\end{array}$ \\
\hline $\begin{array}{l}\text { Solicitação de materiais } \\
\text { gerenciamento de recursos }\end{array}$ & $\begin{array}{l}\text { Materiais de curativo e materiais de escritório; } \\
\text { Avaliação da quantidade de creme disponível no ambulatório e critérios para } \\
\text { distribuição. }\end{array}$ \\
\hline $\begin{array}{l}\text { Gerenciamento } \\
\text { desenvolvidas }\end{array}$ & $\begin{array}{l}\text { Preenchimento de tabelas específicas de registro dos pacientes do ambulatório no } \\
\text { setor: } \\
\text { - Tabela de registo do paciente no ambulatório; } \\
\text { - Tabela de dispensação do creme para prevenção e tratamento da Síndrome } \\
\text { - Aão-Pé; } \\
\text { Atendimento no ambulatório; } \\
\text { Consulta de enfermagem ao paciente em condição de internação hospitalar; } \\
\text { Teleatendimento; } \\
\text { Preenchimento da produtividade do setor. }\end{array}$ \\
\hline Resposta a parecer interdisciplinar & Responde e anexa em prontuário parecer encaminhados pela equipe interdisciplinar. \\
\hline $\begin{array}{l}\text { Preenchimento de parecer } \\
\text { interdisciplinar }\end{array}$ & Preenche e encaminha parecer a outros profissionais da equipe interdisciplinar. \\
\hline
\end{tabular}

Fonte: Autores (2020).

\section{Ações Técnicas E Educativas}

Nos momentos de observação participante foi possível identificar as ações técnicas e desenvolvidas pelo enfermeiro responsável pelo ambulatório da Síndrome Mão-Pé. De acordo com o Cofen (2016), as ações técnicas são direcionadas para os cuidados assistenciais diretos e específicos do profissional enfermeiro ao indivíduo, à família e à coletividade, podendo ainda ser constituídas em ambiente hospitalar, ambulatorial ou da promoção e prevenção de saúde, onde o enfermeiro deve ter competência técnica e científica para seu desenvolvimento, bem como planejamento por meio de protocolos e rotinas (Cofen, 2016; Santos et al., 2016).

A análise dos dados do diário de campo também permitiu identificar que dentre as ações desenvolvidas pelo enfermeiro no ambulatório de prevenção e tratamento da Síndrome Mão-Pé, ocorre também o desenvolvimento de ações educativas na consulta de enfermagem. O Ministério da Saúde (2006) caracteriza educação em saúde como: "Processo educativo de construção de conhecimentos em saúde que visa à apropriação temática pela população". No cenário da prática assistencial do profissional 
enfermeiro, a educação em saúde é subsidiada por meio de uma relação diálogo-reflexiva que permeia a conscientização do processo saúde-doença do indivíduo (Brasil, 2006; Falkenberg et al., 2014; Gautério et al., 2013).

Observa-se que ações educativas permeadas pela educação em saúde são implantadas na consulta inicial de enfermagem, na consulta subsequente e no teleatendimento, sendo essa conduta influente para a efetivação do tratamento proposto e implementação do monitoramento mensal do paciente por meio do ambulatório. Buscando apresentar as ações educativas identificadas na consulta de enfermagem foi elaborado o Quadro 2 que segue.

Quadro 2 - Ações técnicas e educativas desenvolvidas pelo Enfermeiro do ambulatório da prevenção e tratamento da Síndrome Mão-Pé.

\begin{tabular}{|c|c|}
\hline \multicolumn{2}{|r|}{ Ações técnicas e educativas } \\
\hline Ação & rística da ação \\
\hline $\begin{array}{l}\text { Exame físico na } \\
\text { Consulta de primeira } \\
\text { vez }\end{array}$ & $\begin{array}{l}\text { Apresentação profissional e escuta ativa; } \\
\text { Investigação minuciosa por meio do exame físico nos quirodáctilos, leito palmar, pododáctilos e leito plantar } \\
\text { na consulta de primeira vez: } \\
\text { - } \quad \text { Descamação prévia } \\
\text { - } \quad \text { Hidratação da pele } \\
\text { Registro do exame físico em livro próprio do setor. }\end{array}$ \\
\hline $\begin{array}{l}\text { Exame físico na } \\
\text { Consulta subsequente }\end{array}$ & $\begin{array}{l}\text { Avaliação do registro do exame físico da última consulta no ambulatório em livro próprio do setor; } \\
\text { Acareação dos dados subjetivos: Frequência, intensidade e extensão dos sintomas; } \\
\text { Investigação minuciosa por meio do exame físico de: } \\
\text { - } \quad \text { Dor } \\
\text { - } \quad \text { Sensibilidade } \\
\text { - } \quad \text { Eritema } \\
\text { - } \quad \text { Descamação (seca ou úmida) } \\
\text { - } \quad \text { Ressecamento } \\
\text { - Parestesia } \\
\text { - } \quad \text { Disestesia } \\
\text { - Hiperceratose (espessamento cutâneo) } \\
\text { - Nível de hidratação } \\
\text { Registro da situação clínica atual em livro próprio do setor. }\end{array}$ \\
\hline $\begin{array}{l}\text { Orientações } \\
\text { Enfermagem }\end{array}$ & $\begin{array}{l}\text { Esclarecimento da terapia antineoplásica oral Capecitabina, contraindicações durante o tratamento, efeitos } \\
\text { colaterais e sintomas para retorno imediato à emergência; } \\
\text { Orientação sobre a importância da profilaxia e da adesão ao tratamento proposto; } \\
\text { Resposta a questionamentos emergidos durante a consulta. }\end{array}$ \\
\hline $\begin{array}{l}\text { Capacitação de } \\
\text { pacientes e familiares }\end{array}$ & $\begin{array}{l}\text { Capacitação para reconhecimento prévio dos sintomas da Síndrome Mão-Pé, o impacto causado pela mesma } \\
\text { no autocuidado e contraindicações durante o tratamento; } \\
\text { Estratégias para prevenção e manejo da síndrome por meio da utilização do creme para prevenção e tratamento } \\
\text { da Síndrome Mão-Pé; } \\
\text { Fornecimento de orientações impressas ao final da consulta. }\end{array}$ \\
\hline Teleatendimento & $\begin{array}{l}\text { Avaliação de critérios mínimos para efetivação desse molde de atendimento; } \\
\text { Avaliação de registro do paciente no setor enquadrado nesse tipo de atendimento. }\end{array}$ \\
\hline
\end{tabular}




\section{Discussão}

A análise dos estudos permitiu identificar que as ações desenvolvidas pelos enfermeiros, no contexto de cuidado ambulatorial para prevenção e manejo da Síndrome Mão-Pé em paciente oncológicos, estão intimamente relacionadas à identificação do fator de risco, já que o paciente oncológico apresenta suscetibilidade advinda do tratamento com o quimioterápico oral Capecitabina, à avaliação clínica e diagnóstica durante a anamnese, à avaliação diária da pele e das condições do paciente, a fim de capacitar o binômio paciente e familiar sobre os sinais e sintomas referentes ao desenvolvimento da SMP, bem como a utilização profilática de creme de hidratação profunda e da proposta de educação em saúde como estratégia do cuidado, a fim de reduzir danos a essa população.

Frente ao exposto, para a síntese desta revisão, emergiram duas categorias de ações desenvolvidas pelo enfermeiro: Categoria I - Ações administrativas e Categoria II - Ações técnicas e educativas, bem como a identificação do fluxo de atendimento.

\section{Fluxo de atendimento ao paciente acometido pela Síndrome Mão-Pé}

O fluxo seguido pelos pacientes atendidos no Ambulatório de prevenção e tratamento da Síndrome Mão-Pé (ASMP) permite que, a maior parte destes, em uso de fármacos quimioterápicos orais com predisposição ao desenvolvimento da SMP, sejam encaminhados ao atendimento no ambulatório precocemente.

Cabe ressaltar a ocorrência de extemporaneidade do fluxo institucional que aumenta a necessidade do atendimento ambulatorial dos pacientes. Identificou-se que por vezes, o encaminhamento destes pacientes é feito por outros profissionais, que não os médicos prescritores, como ocorre, por exemplo com os enfermeiros da central de quimioterapia e radioterapia que, ao identificarem o paciente em uso de fármacos quimioterápicos orais com predisposição ao desenvolvimento da SMP, e que não estejam em acompanhamento pelo ASMP, fazem o encaminhamento deste.

A análise dos resultados permitiu identificar que as ações desenvolvidas pelo enfermeiro do ASMP são reconhecidas como benéficas aos pacientes pelos outros componentes da equipe de saúde. Para Son et al. (2009), a participação ativa da equipe multidisciplinar ao paciente oncológico é um elemento-chave no gerenciamento eficaz da síndrome mão-pé. Destaca-se ainda, que a compreensão do processo de trabalho do profissional enfermeiro no ASMP por outros membros da equipe favorecem para a integralidade das ações de saúde.

Simão et al. (2012) evidencia que a severidade dos sintomas está relacionada principalmente à dose-cumulativa do quimioterápico oral, intensificando-se a cada novo ciclo da droga antineoplásica. Dessa forma, é de suma importância uma abordagem iniciada precocemente pelo profissional enfermeiro por meio de um cuidado sistematizado, favorecendo a otimização dos resultados. Saif (2011) reafirma a necessidade de uma abordagem precoce, de caráter educativo para prevenção ou retardo dessa toxicidade, sugerindo ainda o desenvolvimento de programas que facilitem a adesão ao tratamento, especificamente na identificação e gerenciamento precoce dos efeitos colaterais.

\section{Categoria I: Ações administrativas}

No que diz respeito às ações administrativas realizadas pelo enfermeiro, a análise dos dados deste estudo oportunizou evidenciar que, neste cenário, o maior quantitativo de ações desenvolvidas pelo profissional enfermeiro responsável pelo ASMP é de caráter administrativo. Cabe ressaltar que as ações administrativas vão desde a identificação destes pacientes no ASMP até a manutenção rigorosa por meio do monitoramento dos pacientes e registros quanto à condição clínica e tissular referente ao risco de desenvolvimento da SMP destes pacientes e manejo da síndrome. Todas estas ações demandam grande parte do tempo de trabalho deste profissional, podendo resultar em uma sobrecarga profissional, o que corrobora com Costa et al. (2018) que 
evidencia o acúmulo de tarefas, a redução do quantitativo de profissionais e o quantidade de atendimento fornecido nas unidades como fatores que podem propiciar uma carga excessiva de trabalho influenciando em um distanciamento da assistência à saúde.

Dentre as ações administrativas identificadas, destaca-se a dispensação do creme de hidratação profunda para prevenção e/ou tratamento da Síndrome Mão-Pé realizado nas consultas subsequentes, corroborando com Webster-Gandy, How e Harrold (2007) e Son et al. (2009), que consideram a aplicação regular do creme emoliente para a hidratação da pele como manejo preventivo da síndrome, favorecendo o retardo da toxicidade. Para que ocorra a dispensação deste creme é importante avaliar alguns critérios como: condição socioeconômica, performance status, residir distante do instituto, dentre outros fatores.

\section{Categoria II: Ações técnicas e educativas}

As ações administrativas são primordiais para o desenvolvimento das ações técnicas e educativas. Cabe ressaltar que dentre as ações técnicas desenvolvidas pelo enfermeiro no ambulatório de prevenção e tratamento da Síndrome Mão-Pé, ocorre também o desenvolvimento de ações educativas implantadas na consulta inicial de enfermagem, na consulta subsequente e no teleatendimento, sendo essa conduta influente para efetivação do tratamento proposto e implementação do monitoramento mensal do paciente, por meio do ambulatório.

Neste estudo, evidenciou-se que a principal ação desenvolvida pelo profissional enfermeiro responsável pelo ASMP é a consulta de enfermagem, sendo esta o principal instrumento de cuidado, cujos objetivos incluem prevenção, tratamento e controle da síndrome, por meio da dispensação de creme de hidratação profunda, orientações de enfermagem e valorização do cuidado compartilhado, que segundo Costa et al. (2018) favorece em um importante processo de interação e integração com o paciente sob a ótica da educação em saúde.

As consultas de primeira vez ocorridas no ASMP demandam mais tempo, com duração média de 45 minutos. Nestas, observa-se o foco em um atendimento humanizado e o estabelecimento de vínculo entre o profissional, os pacientes e os familiares. Trata-se de um recurso terapêutico que favorece a integração da equipe de saúde em prol do cuidado e do tratamento para além da patologia, levando em consideração a história de quem está sendo cuidado, conforme preconiza a Política Nacional de Humanização (Brasil, 2010).

Identifica-se que a CE oportuniza o fornecimento de informações específicas a esses pacientes sobre a terapia com Capecitabina, orienta e capacita para o reconhecimento dos primeiros sinais e sintomas da SMP. Esta ação corrobora com o estudo desenvolvido por Webster-Gandy, How e Harrold (2007) que considera imprescindível a capacitação dos pacientes para reconhecimento prévio da síndrome, e ciência do grau de gravidade com necessidade de intervenção, sendo fundamental a consulta de enfermagem antes do início do tratamento para que ocorra a estratégia de educação em saúde, com a contribuição do tempo necessário para discussão e compreensão de todos os aspectos acerca do tratamento, favorecendo a adesão.

Dentre a pluralidade de ações desenvolvidas pelo enfermeiro durante a consulta de enfermagem, evidencia-se o esclarecimento da terapia antineoplásica oral, o risco de desenvolvimento da eritrodisestesia palmo-plantar, a capacitação para identificação prévia dos sintomas da Síndrome Mão-Pé, dentre outros possíveis efeitos colaterais, bem como estratégias para prevenção e manejo da síndrome (Son et al., 2009; Webster-Gandy, How, \& Harrold, 2007).

Nas consultas subsequentes no ASMP ocorre a investigação dos sinais e sintomas da síndrome por meio da escuta ativa, anamnese e exame físico, integrante do Processo de Enfermagem (PE), que oportuniza a avaliação da adesão ao tratamento proposto por meio do acompanhamento mensal. Lassere e Hoff (2004) apontam a supervisão dos pacientes como estratégia para identificar precocemente sinais de toxicidade que possibilitam intervenções adequadas, bem como o manejo apropriado da síndrome quando já estabelecida. Nesse contexto, a consulta de enfermagem é um importante instrumento não apenas para avaliar, mas para constituir uma assistência integral e holística embasada na proposta de educação em saúde. 
Por vezes o acompanhamento dos pacientes atendidos no ASMP é dificultado quando há indicação clínica de internação destes pacientes. Nestes casos, o enfermeiro responsável pelo ASMP estende seu acompanhamento do ASMP para o local de internação do paciente, visando garantir a continuidade deste atendimento, apontado como imprescindível para intervenções e manejo adequado da síndrome (LasserE \& Hoff, 2004).

Evidenciou-se que a CE em seus diversos modelos desenvolvidos no ASMP permite o monitoramento dos pacientes com potencial risco de desenvolvimento desta síndrome, sendo um método eficaz de gerenciamento desta, o que corrobora com Simão et al. (2012) e Webster-Gandy, How e Harrold (2007), em que se reafirma que se os sintomas não forem gerenciados, eles podem progredir rapidamente, tornando dessa forma necessária a ingerência de intervenções caras e agressivas.

Buscando favorecer a ocorrência e a manutenção da adesão ao tratamento, é desenvolvida a consulta subsequente no molde de teleatendimento frente à demanda específica de saúde que impossibilite o comparecimento ao ambulatório, corroborando com Webster-Gandy, How e Harrold (2007), que enfatizam a importância e a necessidade do gerenciamento telefônico e a triagem durante os primeiros ciclos de terapia. Ferreira et al. (2017) considera que o contato telefônico possibilita estratégias de educação em saúde, enfrentamento e manejo dos efeitos adversos, bem como um espaço para oferecer meios de enfrentamento ao indivíduo.

Moretto, Contim e Santo (2019) ressaltam que o teleatendimento é uma modalidade assistencial em ascendimento que viabiliza a interação ativa entre profissionais de saúde e pacientes, tendo como principal fator interveniente a redução de custos, com a assistência prestada, fatores epidemiológicos e barreiras territoriais, sendo incumbência do enfermeiro oncológico o papel essencial de educação em saúde frente ao atendimento domiciliar, bem como de avaliação da aderência ao tratamento para garantir que os pacientes estejam realizando os regimes de dosagem de maneira correta e relatando os efeitos colaterais precisamente frente ao tratamento domiciliar (Gerbrecht, 2003; Marsé et al., 2004; Moretto, Contim, \& Santo, 2019).

\section{Conclusão}

Com base nos resultados do estudo, identificou-se que existe um fluxo que é seguido pelos pacientes e que este oportuniza o acompanhamento dos pacientes em uso do antineoplásico oral Capecitabina em sua quase totalidade.

Foi possível ainda identificar diferentes ações desenvolvidas pelo profissional enfermeiro responsável pelo ambulatório de prevenção e tratamento da Síndrome Mão-Pé. As ações foram organizadas em duas categorias, denominadas: ações administrativas e ações técnicas e educativas, implementadas durante as consultas de enfermagem.

A consulta de enfermagem caracterizou a principal ação educativa desenvolvida neste ambulatório e é operacionalizada com intuito preventivo quanto a instauração da síndrome além de manejo e controle da mesma. Nota-se um importante processo de interação e estabelecimento de vínculo com o paciente, utilizando-se a educação em saúde como a principal estratégia de cuidado, associado à valorização do cuidado compartilhado, contribuindo para adesão ao tratamento por meio do estímulo ao comprometimento no tratamento e no autocuidado.

As ações administrativas são as que demandam maior parte do tempo do profissional enfermeiro responsável pelo ambulatório da Síndrome Mão-Pé. Por meio desta, foi possível evidenciar que ocorre aderência ao acompanhamento oferecido neste ambulatório, pois há um quantitativo mínimo de absenteísmos nas consultas agendadas. Outro fato que aponta a importância deste ambulatório para os pacientes é que, quando há necessidade de internação hospitalar por intercorrências clínicas, há uma preocupação por parte dos pacientes e familiares que fazem contato telefônico informando a ocorrência e justificando o seu não comparecimento à consulta agendada.

Frente ao exposto, sob a ótica do desenvolvimento do cuidado integral em todas as esferas de saúde, a proposta deste estudo foi analisar as ações desenvolvidas pelo profissional enfermeiro em um ambulatório de prevenção e tratamento da Síndrome Mão-Pé induzida pelo uso de Capecitabina. Pode-se inferir que o papel do profissional enfermeiro em um ambulatório 
especializado e contemporâneo impacta positivamente na qualidade de vida dos pacientes atendidos, subsidiando meios para adesão ao tratamento e autonomia dos pacientes através da estruturação do cuidado que possibilita a integralidade e a longitudinalidade das ações de saúde.

De acordo com a revisão de literatura realizada para elaboração desse estudo, foi possível perceber a escassez de publicações relacionada a temática. Dessa forma, destaca-se a importância de futuras investigações sobre essa temática, bem como outras dimensões do processo de cuidado do paciente em uso de terapia com antineoplásicos orais assistido em ambulatório especializado.

\section{Referências}

Barros, A. L. B. L., Sanchez, C. G., Lopes, J. L., Dell’Acqua, M. C. Q., Lopes, M. H. B. M., \& Silva, R. C. G. (2015). Processo de enfermagem: guia para prática/ Conselho Regional de Enfermagem de São Paulo. https://portal.coren-sp.gov.br/sites/default/files/SAE-web.pdf.

Brasil (2019). Ministério da Saúde. Instituto Nacional do Câncer. Estimativa 2020: Incidência do câncer no Brasil. INCA. https://www.inca.gov.br/sites/ufu.sti.inca.local/files//media/document//estimativa-2020-incidencia-de-cancer-no-brasil.pdf.

Brasil (2019). Ministério da Saúde. Instituto Nacional do Câncer. ABC do câncer: abordagens básicas para o controle do câncer. (5a ed.), INCA.

Brasil (2018). Ministério da Saúde. Instituto Nacional do Câncer. Estimativa 2016: Incidência do câncer no Brasil. INCA. https://www.inca.gov.br/en/node/2396.

Brasil (2016). Ministério da Saúde. Secretaria de Gestão do Trabalho e da Educação na Saúde. Departamento de Gestão e da Regulação do Trabalho em Saúde. Câmara de Regulação do Trabalho em Saúde. 2006.

Brasil (2010). Ministério da Saúde. Secretaria de Atenção à Saúde. Núcleo Técnico da Política Nacional de Humanização. HumanizaSUS: Documento base para gestores e trabalhadores do SUS. (4a ed.).

Canonici, E. L. (2014). Modelos de unidades e serviços para organização da atenção ambulatorial especializada em Sistemas Regionais de Atenção à Saúde. Proadi-Sus; 2014.

Capecitabina (2020). São Paulo: Eurofarma laboratórios S.A. Bula de remédio.

Conselho Federal de Enfermagem - COFEN (2016). Resolução no 509, de 15 de março de 2016. Atualiza a norma técnica para Anotação de Responsabilidade Técnica pelo Serviço de Enfermagem e define as atribuições do enfermeiro responsável técnico. http://www.cofen.gov.br/resolucao-cofen-no-050920162_39205.html.

Conselho Federal de Enfermagem - COFEN (2009). Resolução n.358, de 15 de outubro de 2009. Dispõe sobre a Sistematização da Assistência de Enfermagem e a implementação do Processo de Enfermagem em ambientes, públicos ou privados. http://novo.portalcofen.gov.br/resoluo-cofen3582009_4384.html.

Conselho Nacional de Saúde CNS (2016). Resolução $n^{o}$ 510, de 7 de abril de 2016. Diretrizes e normas regulamentadoras de pesquisas envolvendo seres humanos.

Costa, C. S., Normann, K. A. S., Tanaka, A. K. S. R. \& Cicolella, D. A. (2018). Influência da sobrecarga de trabalho do enfermeiro na qualidade da assistência. Revista Uningá, Maringá, 4(55), 110-120. http://revista.uninga.br/index.php/uninga/article/view/2403/1796.

Demoliner, L. P. \& Corte, T. W. F. (2008). Atenção farmacêutica para pacientes usuários de Lapatinibe. Trabalho de conclusão de curso, Faculdade de Farmácia da Pontifícia Universidade Católica do Rio Grande do Sul.

European Medicines Agency - EMA \& Scienc Medicines Health - SMH. Resumo do EPAR destinado ao público: Xeloda. http://www.ema.europa.eu/ema/index.jsp?curl=pages/medicines/human/medicines/000316/human_med_001157.jsp\&mid=WC0b01ac058001d124.

Falkenberg, M. B., Mendes, T. P. L., Moraes, E. P., \& Souza, E. M. (2014). Educação em saúde e educação na saúde: conceitos e implicações para a saúde coletiva. Ciência \& Saúde Coletiva, 19(3), 847-852. FapUNIFESP (SciELO). https://www.scielosp.org/article/csc/2014.v19n3/847-852.

Ferreira, E. B., Kamada, I., Reis, P. E. D., Cruz, F. O. A. M., Jesus, C. A. C. \& Pinho, D. L. M. (2017). Contato telefônico como estratégia para a promoção de conforto ao paciente submetido à quimioterapia. Rev. EnfermUfpe, 11(5), 1936-1942. https://periodicos.ufpe.br/revistas/revistaenfermagem/article/view/23343.

Gautério, D.P., Vidal, D. A. S., Barlem, J. G. T., \& Santos, S. S. C. (2013). Ações educativas do enfermeiro para a pessoa idosa: estratégia saúde da família. Rev. Enferm. Uerj, 21(2), 824-828.

Gerbrecht, B. M. (2003). Current Canadian experiencewithcapecitabine: partneringwithpatientstooptimizetherapy. CancerNurs, 26(2), 161-157. https://pubmed.ncbi.nlm.nih.gov/12660565/.

Júnior, W. B., Tomaz, A. C. A., Vieira, L. F. A., Silva, P. M. X., Oliveira, S. M. B., \& Lima, M. K. S. (2017). Síndrome mão-pé induzida por Capecitabina: Relato de caso. CogitareEnferm. 22(1): 01-04.

Lassere, Y \& Hoff, P. (2004). Management ofhand-footsyndrome in patientstreatedwithcapecitabine (Xeloda®). European Journal of Oncology Nursing, 8. https://pubmed.ncbi.nlm.nih.gov/15341880/.

Marconi, M. A. \& Lakatos, E. M. (2017). Fundamentos de metodologia científica. (8a ed.), Atlas. 
Research, Society and Development, v. 10, n. 4, e49910414383, 2021

(CC BY 4.0) | ISSN 2525-3409 | DOI: http://dx.doi.org/10.33448/rsd-v10i4.14383

Marsé, H., Cutsem, E. V., Grothey, A., \& Valverde, S. (2004). Management of adverse event sando ther practical considerations in patients. Eur J OncolNurs, 8(1), 16-30. https://pubmed.ncbi.nlm.nih.gov/15341879/.

Moretto, I. G., Contim, C. L. V. \& Santo, F. H. E. (2019). Acompanhamento por telefone como intervenção de enfermagem a pacientes em quimioterapia ambulatorial: revisão integrativa. Rev. Gaúcha Enferm. 40. https://doi. org/10.1590/1983-1447.2019.20190039.

Oliveira, A. T. \& Queiroz, A. P. A. (2012). Perfil de uso de terapia antineoplásica oral: A importância da orientação farmacêutica. RevBras Farm. HospServ Saúde. 3(4), 24-29.

Santos, K. C., Fonseca, D. F., Oliveira, P. P., Duarte, A. G. S., Melo, J. M. A. \& Souza, R. S. (2020). Atenção à saúde do homem: construção e validação de instrumento para consulta de enfermagem. RevBras Enferm. 73(3):e20190013.

Santos, I. M. F., Fontes, N. C. F., Silva, R. S., \& Brito, S. S. J. (2016). Sistematização da assistência de enfermagem: Guia prático. Salvador: COREN - BA, p. 40. http://ba.corens.portalcofen.gov.br/wpcontent/uploads/2016/07/GUIA_PRATICO_148X210_COREN.pdf.

Saif, MW. (2011). Capecitabine And Hand-footsyndrome. Expert Opin. DrugSaf, 10(2), 159-169. https://pubmed.ncbi.nlm.nih.gov/21174613/.

Sociedade Brasileira de Farmacêuticos em Oncologia - SOBRAFO. (2011). Guia para notificação de reações adversas em oncologia. http://portal.anvisa.gov.br/documents/33868/2893744/Guia+para+Notifica\%C3\%A7\%C3\%A3o+de+Rea\%C3\%A7\%C3\%B5es+Adversas+em+Oncologia/64d 680f5-1b77-4ee6-b4c8-88b3ee3f4edf.

Son, H. S., Lee, W. Y., Lee, W. S., Yun, S. H. \& Chun, H. K. (2009). ComplianceandEffective Management ofthe Hand-FootSyndrome in ColonCancerPatientsReceivingCapecitabine as AdjuvantChemotherapy. Yonsei Medical Journal, 50(6), 796-802. Yonsei University Collegeof Medicine. http://dx.doi.org/10.3349/ymj.2009.50.6.796.

Simão, D. A. S., Lima, E. D. R. P., Souza, R. S., Faria, T. V., \& Azevedo, G.F. (2012). Síndrome mão-pé induzida por quimioterapia: relato de um caso. RevBrasEnferm, 65(2), 374-378. https://www.scielo.br/pdf/reben/v65n2/v65n2a26.pdf.

Vaghetti, H., Reis, D., Kerber, N. C., Azambuja, E. \& Fernandes, G. (2004). Percepções dos enfermeiros acerca das ações administrativas em seu processo de trabalho. Rev.BrasEnferm, 57(8), 316-320. https://www.scielo.br/scielo.php?pid=S0034-71672004000300012\&script=sci_abstract\&tlng=pt.

Webster-Gandy, J. D., How, C. \& Harrold, K. (2007). Palmar-plantar erythrodysesthesia (PPE): a literature review withcommentaryexperience in a cancer centre. European Journal Of Oncology Nursing, 11(3), 238-246. http://dx.doi.org/10.1016/j.ejon.2006.10.004. 